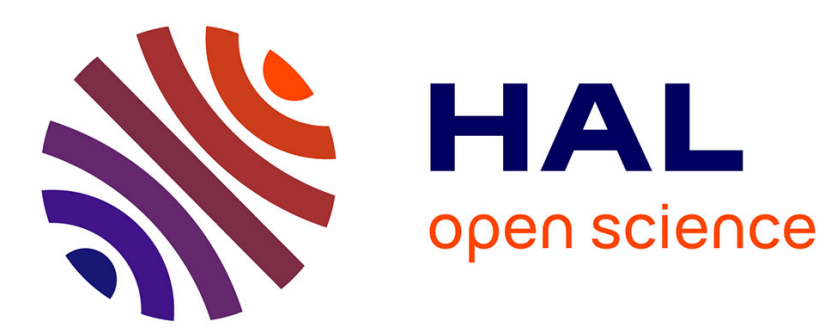

\title{
Visual Servoing through mirror reflection
}

Eric Marchand, François Chaumette

\section{To cite this version:}

Eric Marchand, François Chaumette. Visual Servoing through mirror reflection. ICRA'17 - IEEE International Conference on Robotics and Automation, May 2017, Singapore, Singapore. hal$01445484 \mathrm{v} 3$

\section{HAL Id: hal-01445484 \\ https://hal.inria.fr/hal-01445484v3}

Submitted on 26 Jan 2017

HAL is a multi-disciplinary open access archive for the deposit and dissemination of scientific research documents, whether they are published or not. The documents may come from teaching and research institutions in France or abroad, or from public or private research centers.
L'archive ouverte pluridisciplinaire HAL, est destinée au dépôt et à la diffusion de documents scientifiques de niveau recherche, publiés ou non, émanant des établissements d'enseignement et de recherche français ou étrangers, des laboratoires publics ou privés. 


\title{
Visual Servoing through mirror reflection
}

\author{
Eric Marchand, François Chaumette
}

\begin{abstract}
Apart the use of catadioptric cameras, only few visual servoing works exploit the use of mirror. Such a configuration is however interesting since it allows to overpass the limited camera field of view. Based on the known projection equations involved in such a system, this paper introduces the theoretical background that allows the use of planar mirror for visual servoing in different configurations. Limitations intrinsic to such systems, such as the number of d.o.f actually controllable, is then discussed. Experiments using a mirror mounted on the end-effector of a 6 d.o.f robot validate the proposed approach.
\end{abstract}

\section{INTRODUCTION}

Visual servoing [3] has proved to be a very efficient way to control robot using information provided by one or multiple cameras. Vision sensors have the main advantage to get a rich information of the robot environment. However standard perspective cameras have a limited field of view. Considering mirrors may extend the camera field of view. This is usually achieved with catadioptric camera [13] or with controlled mirror such as in the recent Saccade robot [6]. This paper deals with the combination of visual servoing and a planar mirror. Although coupling visual servoing and mirror within catadioptric system (omnidirectional camera) has been seen as a powerful option, few research papers consider the use of mirrors in visual servoing when the relative position between the camera and mirror varies.

Most of the literature in this area is related to the use of catadioptric camera e.g. [2][13][5]. In such systems, the camera and the mirror are rigidly attached and the catadioptric system is mounted on the robot. The main difference between such system and a classical visual servoing system is the projection model (central projection model) and consequently the interaction matrix. The resulting control has also proved to feature good decoupling properties for some particular visual features [18].

The use of planar mirrors has been considered to simulate multi-camera system. Indeed, with a mirror, it is possible to obtain a stereo view using a single camera (planar catadioptric stereo [14]). Although such a system may allow to provide useful information for robot control, the mirrors positions are not controlled. Multiple stationary planar mirrors systems for micro alignment of optical fiber using a visual servoing techniques was also reported in [15]. The control of the alignment process is achieved in closed loop using the projection and reflection of the optical fiber on both mirrors.

\footnotetext{
Eric Marchand is with Université de Rennes 1, IRISA, Inria Rennes, Rennes, France Eric.Marchandeirisa.fr. François Chaumette is with Inria, IRISA, Rennes, France Francois. Chaumettedinria.fr
}

Such visual servoing approach avoid precise calibration of the mirror-based stereo system.

A more classical visual servoing system is reported in [9][8] where the motion of the robot end-effector is controlled through its reflection in a mirror. Let us note that in this work, the mirror and the camera are stationary. We will see that such a system is in fact very similar to a classical visual servoing structure.

In all previous works, the mirrors are motionless and the relative pose between the camera and the mirror(s) is fixed. Few works considered mobile mirrors or a varying mirror/camera position. Laser steering application for microsurgery is reported in [19], [1]. A laser spot controlled thanks to the motion of a 2 d.o.f mirror is achieved using a stereo visual servoing approach. In the context of high speed visual servoing, some systems that consider mirrors, have been proposed to achieve high-speed gaze control: Saccade mirror [7], [6]. These systems consider two [7] or three [6] one d.o.f mirror to provide fast tracking capabilities $(1000 \mathrm{~Hz})$ of moving target. The proposed Saccade Mirror system allows to simulate a pan/tilt camera while allowing very fast saccade and increasing the field of view of the camera. Open loop control law is considered for the tracking process.

In this paper we provide the theoretical background required for the development of closed-loop visual servoing. We consider the three possible configurations which may allow to either control a moving camera, a moving target attached to the robot or a moving mirror. In all these cases the target is observed by the camera through its reflection in the mirror. After recalling the reflection and the resulting projection equations, we will see that the two former configurations can be easily formulated using classical visual servoing control law. The last case, that is controlling the mirror, is the key contribution of this paper. We will exhibit the interaction matrix that links the motion of a point in the image to the motion of the mirror. We will also demonstrate that in practice only 3 mirror d.o.f. are actually controllable. We will thus propose to use the remaining d.o.f. to cope with the issue induced by the fact that the mirror has not an infinite size. Experiments using a mirror mounted on the end-effector of a 6 d.o.f robot validates the proposed approaches.

\section{REFLECTION AND PROJECTION MODELING}

Let us define the mirror frame such that $\mathrm{x}$ and $\mathrm{y}$ axes are in the mirror plane $\Pi$ and $\mathrm{z}$ axis is perpendicular to the mirror (see Figure 1). The equation of the mirror in this frame is given by:

$$
{ }^{m} \mathbf{n}^{\top m} \mathbf{X}=0
$$


where ${ }^{m} \mathbf{n}=\left(\begin{array}{lll}0 & 0 & 1\end{array}\right)^{\top}$ is the normal to the mirror $\boldsymbol{\Pi}$ expressed in the mirror frame. Let ${ }^{m} \mathbf{X}_{R}$ be the symmetric of the point ${ }^{m} \mathbf{X}$ wrt the mirror. ${ }^{m} \mathbf{X}_{R}$ coordinates are given by (eg, [14]):

$$
{ }^{m} \mathbf{X}_{R}=\left(\mathbf{I}-2^{m} \mathbf{n}^{m} \mathbf{n}^{\top}\right)^{m} \mathbf{X}
$$

Note that this equation is true whatever the choice of ${ }^{m} \mathbf{n}$ (subject that $\left\|^{m} \mathbf{n}\right\|=1$ ) if the origin of $\mathcal{F}_{m}$ belongs to plane $\Pi$.

Let ${ }^{c} \mathbf{X}$ be the coordinates of a point in the camera frame $\mathcal{F}_{c}$. Using classical notations for rotation and translation,we have

$$
{ }^{c} \mathbf{X}={ }^{c} \mathbf{R}_{m}{ }^{m} \mathbf{X}+{ }^{c} \mathbf{t}_{m} .
$$

Let

$$
\mathbf{n}^{\top c} \mathbf{X}=d
$$

be the equation of the mirror plane in the camera frame where $\mathbf{n}$ is the unit vector that represents the normal to the plane in the camera frame and $d$ is the orthogonal distance between the camera and the plane (see Figure 1). We thus have $\mathbf{n}={ }^{c} \mathbf{R}_{m}{ }^{m} \mathbf{n}$ and ${ }^{c} \mathbf{t}_{m}=d \mathbf{n}$.

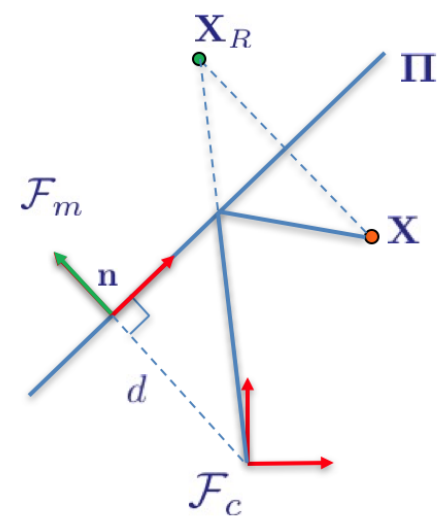

Fig. 1. Reflection and projection with a planar mirror

The coordinates of the reflection ${ }^{c} \mathbf{X}_{R}$ of ${ }^{c} \mathbf{X}$ wrt. the mirror is given by [14] (see proof in Appendix A):

$$
{ }^{c} \mathbf{X}_{R}=\left(\mathbf{I}-2 \mathbf{n n}{ }^{\top}\right)^{c} \mathbf{X}+2 d \mathbf{n}
$$

Finally, the projection $\mathbf{x}$ of ${ }^{c} \mathbf{X}_{R}$ in the image plane is given by:

$$
\mathbf{x}={ }^{c} \mathbf{X}_{R} /{ }^{c} Z_{R}
$$

where ${ }^{c} Z_{R}$ is the third component of ${ }^{c} \mathbf{X}_{R}$.

\section{VISUAL SERVOING THROUGH MIRROR}

Let us consider a basic visual servoing that is specified by observing a set of points $\mathbf{x}_{i}$ at given positions in the image $\mathbf{x}_{i}^{*}$. Thus we want to minimize the norm of the error e defined by:

$$
\mathbf{e}=\left(\begin{array}{c}
\vdots \\
\mathbf{x}_{i}-\mathbf{x}_{i}^{*} \\
\vdots
\end{array}\right)
$$

From this quite general formulation of the problem one has to consider three different configurations of the triplet camera, mirror and target:

A the mirror is mounted on the robot end-effector (see Figure 2).

B the camera is mounted on the robot-end-effector (see Figure 4,.

C the target is mounted on the robot-end-effector (see Figure 5).

In the three cases, one wants to observe a target at a given position in the image through its reflection in the mirror. In any case, to design the control law one has to determine the Jacobian $\mathbf{J}_{\mathbf{x}}$ that links the motion of each point in the image to the motion of the end effector in the joint space, that is

$$
\dot{\mathbf{x}}=\mathbf{J}_{\mathbf{x}} \dot{\mathbf{q}}
$$

where $\dot{\mathbf{q}}$ is the robot joint velocity. In that case, considering that we want an exponential decrease of $\mathbf{e}$, the control law is given by:

$$
\dot{\mathbf{q}}=-\lambda \mathbf{J}_{\mathbf{x}}^{+} \mathbf{e}
$$

To determine $\mathbf{J}_{\mathbf{x}}$, one has to derive the relation (the interaction matrix [3]) that links the motion $\dot{x}$ of a point $\mathrm{x}$ to the motion of the mirror (A), the camera (B), or the target $(\mathrm{C})$. This is the purpose of the two next sections.

\section{CONTROLling THE MiRROR POSITION}

This case is actually the most interesting one. In that case the mirror is mounted on the end effector of the robot while the camera is motionless in the scene (see Figure 2).

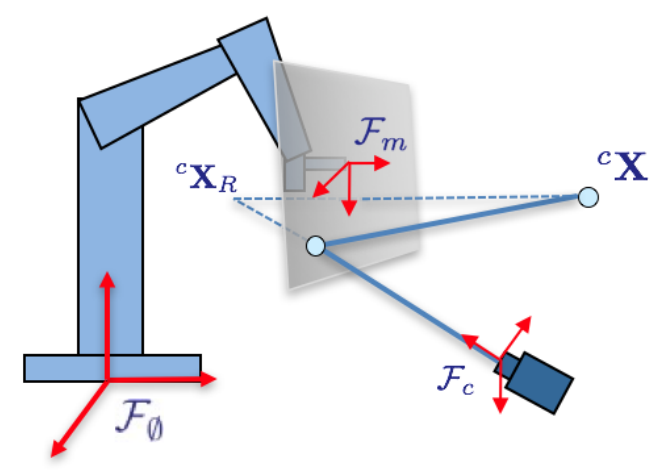

Fig. 2. Case of a controlled mirror

\section{A. Modeling issue and control law}

As already said, one has to define the relation (the interaction matrix) that links the motion $\dot{\mathbf{x}}$ of an image point $\mathbf{x}$ to the motion of the mirror. From equation (6), deriving the normalized coordinates $\mathbf{x}$, we can write:

$$
\dot{\mathbf{x}}=\frac{{ }^{c} \dot{\mathbf{X}}_{R}}{{ }^{c} Z_{R}}-\frac{{ }^{c} \mathbf{X}_{R}}{{ }^{c} Z_{R}^{2}} \dot{Z}_{R}=\frac{{ }^{c} \dot{\mathbf{X}}_{R}}{{ }^{c} Z_{R}}-\frac{\mathbf{x}}{{ }^{c} Z_{R}}{ }^{c} \dot{Z}_{R}
$$

where ${ }^{c} \dot{Z}_{R}$ is the third component of ${ }^{c} \dot{\mathbf{X}}_{R}$. As the main result of this paper, the interaction matrix that links the motion ${ }^{c} \dot{\mathbf{X}}_{R}$ of ${ }^{c} \mathbf{X}_{R}$ to the mirror motion ${ }^{c} \mathbf{v}_{m}$ expressed 
in the camera frame $\left({ }^{c} \mathbf{v}_{m}=\left({ }^{c} \boldsymbol{v}_{m},{ }^{c} \boldsymbol{\omega}_{m}\right)\right.$ where ${ }^{c} \boldsymbol{v}_{m}$ is the mirror translational velocity and ${ }^{c} \boldsymbol{\omega}_{m}$ is the mirror rotational velocity) is given by:

$$
{ }^{c} \dot{\mathbf{X}}_{R}=\left(\begin{array}{cc}
\mathbf{L} \boldsymbol{v} & \mathbf{L} \boldsymbol{\omega}
\end{array}\right)\left(\begin{array}{c}
{ }^{c} \boldsymbol{v}_{m} \\
{ }^{c} \boldsymbol{\omega}_{m}
\end{array}\right)
$$

with

$$
\mathbf{L}_{\boldsymbol{v}}=-2 \mathbf{n} \mathbf{n}^{\top}
$$

and

$$
\mathbf{L} \boldsymbol{\omega}=-2\left(d[\mathbf{n}]_{\times}+\left[{ }^{c} \mathbf{X}_{R}\right]_{\times}[\mathbf{n}]_{\times}^{2}\right)
$$

where $[\mathbf{a}]_{\times}$denotes the skew symmetric matrix associated to the vector $\mathbf{a}$.

Proof. Only mirror is moving, thus, from (5), one can see that

$$
{ }^{c} \dot{\mathbf{X}}_{R}=2 \mathbf{n} \dot{d}+2 \dot{\mathbf{n}} d-2\left(\dot{\mathbf{n}} \mathbf{n}^{\top}+\mathbf{n} \dot{\mathbf{n}}^{\top}\right)^{c} \mathbf{X}
$$

where $\dot{\mathbf{n}}$ and $\dot{d}$ are the motion of the mirror parameters expressed in the camera frame. Since ${ }^{c} \mathbf{X}$ is unknown, it is more convenient to express ${ }^{c} \dot{\mathbf{X}}_{R}$ wrt. ${ }^{c} \mathbf{X}_{R}\left({ }^{c} \mathbf{X}_{R}\right.$ is a priori also unknown, but its projection can be observed and measured in the image, thus only its depth ${ }^{c} Z_{R}$ is in fact unknown). From (5), since ${ }^{c} \mathbf{X}_{R}$ and ${ }^{c} \mathbf{X}$ are symmetric with respect to the mirror plane, we have:

$$
{ }^{c} \mathbf{X}=\left(\mathbf{I}-2 \mathbf{n} \mathbf{n}^{\top}\right){ }^{c} \mathbf{X}_{R}+2 d \mathbf{n}
$$

Injecting (15) in (14), it can be demonstrated that (see proof in appendix B):

$$
{ }^{c} \dot{\mathbf{X}}_{R}=2 \mathbf{n} \dot{d}-2 \dot{\mathbf{n}} d+2\left(\dot{\mathbf{n}} \mathbf{n}^{\top}-\mathbf{n} \dot{\mathbf{n}}^{\top}\right)^{c} \mathbf{X}_{R}
$$

The interaction matrix related to a plane given by (4) is given by [20][16]:

$$
\left(\begin{array}{c}
\dot{\mathbf{n}} \\
\dot{d}
\end{array}\right)=\left(\begin{array}{cc}
\mathbf{0}_{3 \times 3} & {[\mathbf{n}]_{\times}} \\
-\mathbf{n}^{\top} & \mathbf{0}_{1 \times 3}
\end{array}\right)\left(\begin{array}{c}
{ }^{c} \boldsymbol{v}_{m} \\
{ }^{c} \boldsymbol{\omega}_{m}
\end{array}\right)
$$

According to equation (17) we have

$$
2 \mathbf{n} \dot{d}=2 \mathbf{n}\left(\begin{array}{ll}
-\mathbf{n}^{\top} & \mathbf{0}_{1 \times 3}
\end{array}\right)\left(\begin{array}{c}
{ }^{c} \boldsymbol{v}_{m} \\
{ }^{c} \boldsymbol{\omega}_{m}
\end{array}\right)=-2 \mathbf{n} \mathbf{n}^{\top}{ }^{c} \boldsymbol{v}_{m}
$$

and

$$
2 \dot{\mathbf{n}} d=2 d\left(\begin{array}{ll}
\mathbf{0}_{3 \times 3} & {[\mathbf{n}]_{\times}}
\end{array}\right)\left(\begin{array}{c}
{ }^{c} \boldsymbol{v}_{m} \\
{ }^{c} \boldsymbol{\omega}_{m}
\end{array}\right)=2 d[\mathbf{n}]_{\times}{ }^{c} \boldsymbol{\omega}_{m}
$$

and finally, $\dot{\mathbf{n}} \mathbf{n}^{\top}-\mathbf{n} \dot{\mathbf{n}}^{\top}$ being an anti-symmetric matrix, it can be demonstrated that

$$
\begin{aligned}
\dot{\mathbf{n}}{ }^{\top}-\mathbf{n} \dot{\mathbf{n}}^{\top} & =\left[[\mathbf{n}]_{\times} \dot{\mathbf{n}}\right]_{\times}=\left[[\mathbf{n}]_{\times}[\mathbf{n}]_{\times}{ }^{c} \boldsymbol{\omega}_{m}\right]_{\times} \\
& =\left[[\mathbf{n}]_{\times}^{2}{ }^{c} \boldsymbol{\omega}_{m}\right]_{\times}
\end{aligned}
$$

leading to

$$
\begin{aligned}
\left(\dot{\mathbf{n}}{ }^{\top}-\mathbf{n} \dot{\mathbf{n}}^{\top}\right)^{c} \mathbf{X}_{R} & =\left[[\mathbf{n}]_{\times}^{2}{ }^{c} \boldsymbol{\omega}_{m}\right]_{\times}{ }^{c} \mathbf{X}_{R} \\
& =-\left[{ }^{c} \mathbf{X}_{R}\right]_{\times}[\mathbf{n}]_{\times}^{2}{ }^{c} \boldsymbol{\omega}_{m}
\end{aligned}
$$

Considering (18), (19) and (21) in (16) leads to the result given in (12) and (13).
Injecting 11 in 10 leads to the interaction matrix $\mathbf{L}_{\mathbf{x}}$ that is defined by:

$$
\dot{\mathbf{x}}=\mathbf{L}_{\mathbf{x}}\left(\begin{array}{c}
{ }^{c} \boldsymbol{v}_{m} \\
{ }^{c} \boldsymbol{\omega}_{m}
\end{array}\right)
$$

For this configuration, it is convenient to control the motion of the mirror in the mirror frame $\mathcal{F}_{m}$, we thus have:

$$
\dot{\mathbf{x}}=\mathbf{L}_{\mathbf{x}}{ }^{c} \mathbf{V}_{m}\left(\begin{array}{c}
{ }^{m} \boldsymbol{v}_{m} \\
{ }^{m} \boldsymbol{\omega}_{m}
\end{array}\right)
$$

with ${ }^{c} \mathbf{V}_{m}$ the spatial motion transform matrix from the camera frame to the mirror frame ${ }^{1}$. For an eye-to-hand system $\mathbf{J}_{\mathbf{x}}$, introduced in (8), can be expressed as [3]:

$$
\mathbf{J}_{\mathbf{x}}=-\mathbf{L}_{\mathbf{x}}{ }^{c} \mathbf{V}_{m}{ }^{m} \mathbf{V}_{e}{ }^{e} \mathbf{J}(\mathbf{q})
$$

where the classical robot Jacobian ${ }^{e} \mathbf{J}(\mathbf{q})$ is expressed in the end effector frame, ${ }^{m} \mathbf{V}_{e}$ is the spatial motion transform matrix from the mirror frame to the end effector frame. It is a constant matrix as soon as the mirror is rigidly attached to the end effector. Note that, to use 25], the pose of the mirror in the camera frame has to be estimated at each iteration of the control law. Alternatively, one can consider the robot base frame $\mathcal{F}_{\emptyset}$ and the Jacobian given by [3]:

$$
\mathbf{J}_{\mathbf{x}}=-\mathbf{L}_{\mathbf{x}}{ }^{c} \mathbf{V}_{\emptyset}{ }^{\emptyset} \mathbf{V}_{e}{ }^{e} \mathbf{J}(\mathbf{q})
$$

where ${ }^{c} \mathbf{V}_{\emptyset}$ can be computed off-line in a calibration step and ${ }^{\emptyset} \mathbf{V}_{e}$ is obtained thanks to the robot odometry. This latter formulation will be considered in the reported experiments.

\section{B. Actual number of controllable d.o.f}

As expected from such a configuration, motions in the mirror plane (ie, along $\mathrm{x}$ and $\mathrm{y}$ axes and around $\mathrm{z}$ axis of $\mathcal{F}_{m}$ ) do not modify the position of $\mathbf{x}$ in the image (see Figure 3 . When the system is perfectly calibrated first, second and sixth column of $\mathbf{L}_{\mathbf{x}}{ }^{c} \mathbf{V}_{m}$ are equal to zero. That is:

$$
\operatorname{rank} \mathbf{L}_{\mathbf{x}}{ }^{c} \mathbf{V}_{m}=3 \text {. }
$$

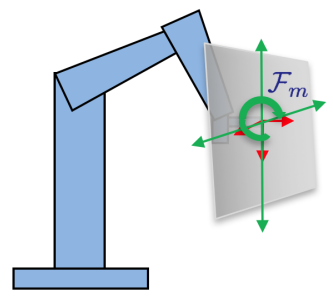

Fig. 3. Invariance of object reflection to in-plane mirror motions

Proof. From $10,, \operatorname{rank} \mathbf{L}_{\mathbf{x}}{ }^{c} \mathbf{V}_{m}=\operatorname{rank}\left(\begin{array}{ll}\mathbf{L}_{\boldsymbol{v}} & \mathbf{L}_{\boldsymbol{\omega}}\end{array}\right)^{c} \mathbf{V}_{m}$ $\left(\begin{array}{cc}\mathbf{L}_{\boldsymbol{v}} & \mathbf{L}_{\boldsymbol{\omega}}\end{array}\right)\left(\begin{array}{cc}{ }^{c} \mathbf{R}_{m} & d[\mathbf{n}]_{\times}{ }^{c} \mathbf{R}_{m} \\ \mathbf{0}_{3 \times 3} & { }^{c} \mathbf{R}_{m}\end{array}\right)=-2\left(\begin{array}{cc}\mathbf{L}_{\mathbf{A}} & \mathbf{L}_{\mathbf{B}}\end{array}\right)$

${ }^{1}$ Spatial transform matrix ${ }^{a} \mathbf{V}_{b}$ allows transforming velocities expressed in frame $\mathcal{F}_{b}$ to velocities expressed in $\mathcal{F}_{a}$. It is given by:

$$
{ }^{a} \mathbf{V}_{b}=\left(\begin{array}{cc}
{ }^{a} \mathbf{R}_{b} & {\left[{ }^{a} \mathbf{t}_{b}\right]_{\times}{ }^{a} \mathbf{R}_{b}} \\
\mathbf{0}_{3 \times 3} & { }^{a} \mathbf{R}_{b}
\end{array}\right) .
$$


with, on one hand,

$$
\mathbf{L}_{\mathbf{A}}=\mathbf{n} \mathbf{n}^{\top c} \mathbf{R}_{m}=\mathbf{n}^{m} \mathbf{n}^{\top}
$$

Since by definition ${ }^{m} \mathbf{n}=\left(\begin{array}{lll}0 & 0 & 1\end{array}\right)^{\top}$ and denoting $\mathbf{n}=$ $\left(n_{1} n_{2} n_{3}\right)^{\top}$

$$
\mathbf{L}_{\mathbf{A}}=\left(\begin{array}{ccc}
0 & 0 & n_{1} \\
0 & 0 & n_{2} \\
0 & 0 & n_{3}
\end{array}\right)
$$

On the other hand, we have:

$$
\begin{aligned}
\mathbf{L}_{\mathbf{B}} & =d \mathbf{n} \mathbf{n}^{\top}[\mathbf{n}]_{\times}{ }^{c} \mathbf{R}_{m}+d[\mathbf{n}]_{\times}{ }^{c} \mathbf{R}_{m}+\left[{ }^{c} \mathbf{X}_{R}\right]_{\times}[\mathbf{n}]_{\times}^{2}{ }^{c} \mathbf{R}_{m} \\
& =\left(d \mathbf{I}_{\mathbf{3}}+\left[{ }^{c} \mathbf{X}_{R}\right]_{\times}[\mathbf{n}]_{\times}\right)[\mathbf{n}]_{\times}{ }^{c} \mathbf{R}_{m} \text { since } \mathbf{n}^{\top}[\mathbf{n}]_{\times}=0 \\
& =\left(d \mathbf{I}_{\mathbf{3}}+\left[{ }^{c} \mathbf{X}_{R}\right]_{\times}[\mathbf{n}]_{\times}\right)^{c} \mathbf{R}_{m}\left[{ }^{m} \mathbf{n}\right]_{\times}
\end{aligned}
$$

since $[\mathbf{n}]_{\times}{ }^{c} \mathbf{R}_{m}={ }^{c} \mathbf{R}_{m}\left[{ }^{c} \mathbf{R}_{m}^{\top} \mathbf{n}\right]_{\times}={ }^{c} \mathbf{R}_{m}\left[{ }^{m} \mathbf{R}_{c} \mathbf{n}\right]_{\times}=$ ${ }^{c} \mathbf{R}_{m}\left[{ }^{m} \mathbf{n}\right]_{\times}$. Then, from 29 ,

$$
\mathbf{L}_{\mathbf{B}}=\left(d^{c} \mathbf{R}_{m}+\left[{ }^{c} \mathbf{X}_{R}\right]_{\times}{ }^{c} \mathbf{R}_{m}\left[{ }^{m} \mathbf{n}\right]_{\times}\right)\left[{ }^{m} \mathbf{n}\right]_{\times}
$$

Expending $\left[{ }^{m} \mathbf{n}\right]_{\times}$with ${ }^{m} \mathbf{n}=\left(\begin{array}{lll}0 & 0 & 1\end{array}\right)^{\top}$ it is immediate to see that the third column of $\mathbf{L}_{\mathbf{B}}$ is $\left(\begin{array}{lll}0 & 0 & 0\end{array}\right)^{\top}$.

Thus in practice, only 3 d.o.f. of the mirror produce to motion in the image (see Figure 3). Nevertheless, the other d.o.f of the mirror can be considered to achieve other tasks such as maintaining the target visible in the mirror.

\section{Using remaining d.o.f to maintain target in the mirror}

Let us consider here that we control the robot in the mirror frame. The Jacobian is then given by 23): $\mathbf{J}_{\mathbf{x}}=-\mathbf{L}_{\mathbf{x}}{ }^{c} \mathbf{V}_{m}$ and the control law is given by:

$$
\mathbf{v}_{m}=-\lambda \mathbf{J}_{\mathbf{x}}^{+} \mathbf{e}
$$

As stated above, rank $\mathbf{J}_{\mathbf{x}}=3$, three dof remain available to achieve other tasks, which can be done using the redundancy framework [3], [4].

$$
\mathbf{v}_{m}=-\lambda \mathbf{J}_{\mathbf{x}}^{+} \mathbf{e}+\mathbf{P}_{\mathbf{x}} \mathbf{J}_{\mathbf{e}_{\mathbf{2}}}^{+} \mathbf{e}_{\mathbf{2}}
$$

with $\mathbf{P}_{\mathbf{x}}=\mathbf{I}-\mathbf{J}_{\mathbf{x}}^{+} \mathbf{J}_{\mathbf{x}}$. Our goal is to maintain the target in the image. Indeed, the mirror is not of infinite size and some motion may imply that the target reflection moves out the limit of the mirror. To maintain the target reflection in the image, a simple way is to control the remaining degrees of freedom of the mirror to maintain the target reflection in the center of the mirror. Let $\overline{\mathbf{x}}_{M}$ be the center of gravity of the mirror and $\overline{\mathbf{x}}$ the center of gravity of the target in the image. The cost function $\mathbf{e}_{\mathbf{2}}$ is then defined as

$$
\mathbf{e}_{2}=\beta\left(\overline{\mathbf{x}}_{M}-\overline{\mathbf{x}}\right)
$$

where $\beta$ is a positive scalar. Note that $\overline{\mathbf{x}}$, being the reflected point, is invariant to the motion of the mirror involved by this secondary task. Indeed $\mathbf{P}_{\mathbf{x}}$ belong to the kernel $\operatorname{Ker} \mathbf{J}_{\mathbf{x}}$. Thus, as demonstrated in the previous paragraph, the motion generated $\mathbf{J}_{\mathbf{e}_{2}}^{+} \mathbf{e}_{2}$ has no effect on the projection of the point in the image. $\overline{\mathbf{x}}$ being invariant, the Jacobian $\mathbf{J}_{\mathbf{e}_{\mathbf{2}}}$ only links the motion of $\overline{\mathbf{x}}_{M}$ to the motion of the mirror. It is given by the classical interaction matrix related to the point $\overline{\mathbf{x}}_{M}$ [3].
With the proposed scheme, target reflection is maintained in the center of the mirror. It is however possible to relax this constraint, for example by allowing the center of the target reflection to be inside an allowed range inside the mirror following the same strategy as in [17].

\section{Simple CASES OF A MOTIONLESS MIRROR}

\section{A. Case of a controlled camera}

In that case the camera is mounted on the end effector of the robot. As can be seen on Figure 4, this is a classical visual servoing configuration [3] with respect to ${ }^{c} \mathbf{X}_{R}$. The corresponding interaction matrix $\mathbf{L}_{\mathbf{x}}$ is thus classically defined by $\dot{\mathbf{x}}=\mathbf{L}_{\mathbf{x}}{ }^{c} \mathbf{v}_{c}$ where ${ }^{c} \mathbf{v}_{c}=\left({ }^{c} \boldsymbol{v}_{c},{ }^{c} \boldsymbol{\omega}_{c}\right)^{\top}$ are the translational and rotational velocity of the camera expressed in the camera frame $\mathcal{F}_{c} . \mathbf{L}_{\mathbf{x}}$ is then given by:

$$
\mathbf{L}_{\mathbf{x}}=\left(\begin{array}{cccccc}
-1 / Z_{R} & 0 & x / Z_{R} & x y & -\left(1+x^{2}\right) & y \\
0 & -1 / Z_{R} & y / Z_{R} & 1+y^{2} & -x y & -x
\end{array}\right)
$$

The proof is given in [10]. It has however to be noted that, here, the visual feature is related to a virtual point $\mathbf{X}_{R}$. Indeed $\mathbf{X}_{R}$ is not a physical point but the symmetric of $\mathbf{X}$ with respect to the mirror. Although the coordinates $\mathbf{x}=\left(\begin{array}{ll}x & y\end{array}\right)^{\top}$ can be directly measured in the image, the depth $Z_{R}$ is obviously unknown but, it can be noted that this is also usually the case for a classical visual servoing process.

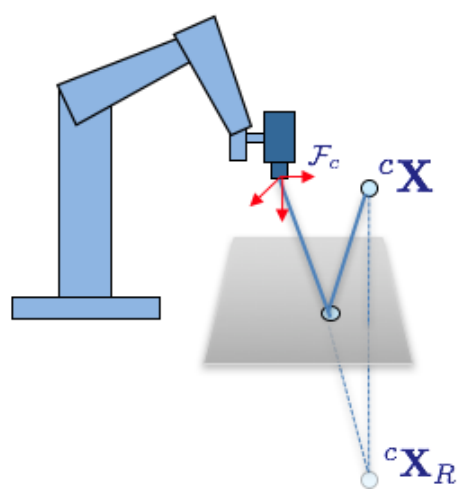

Fig. 4. Case of a controlled camera.

In that case the Jacobian $\mathbf{J}_{\mathbf{x}}$ defined in 9 is given by [3]:

$$
\mathbf{J}_{\mathbf{x}}=\mathbf{L}_{\mathbf{x}}{ }^{c} \mathbf{V}_{e}{ }^{e} \mathbf{J}(\mathbf{q})
$$

where ${ }^{e} \mathbf{J}(\mathbf{q})$ is the classical robot Jacobian expressed in the end effector frame and ${ }^{c} \mathbf{V}_{e}$ is the spatial motion transform matrix from the camera frame to the end effector frame. It is a constant matrix as soon as the camera is rigidly attached to the end effector.

\section{B. Case of a controlled target}

In this last case the mirror and camera are motionless and the target is mounted on the end effector of the robot (see Figure 5). This case was reported in [8][9][15]. 


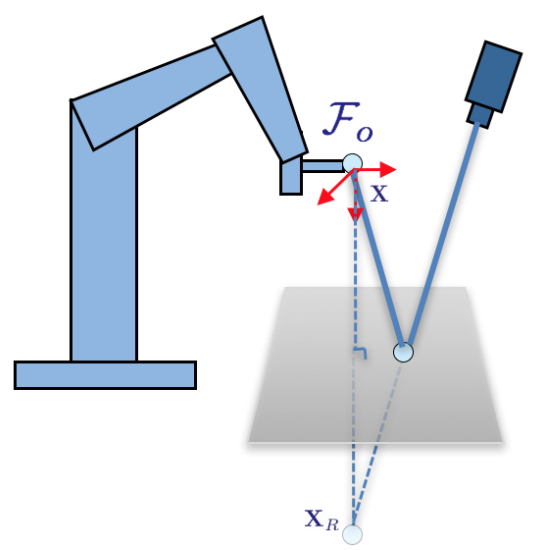

Fig. 5. Case of controlled target.

This case defines in fact a classical eye-to-hand configuration for which the Jacobian $\mathbf{J}_{\mathbf{x}}$ can be expressed as [3]:

$$
\mathbf{J}_{\mathbf{x}}=-\mathbf{L}_{\mathbf{x}}{ }^{o} \mathbf{V}_{e}{ }^{e} \mathbf{J}(\mathbf{q})
$$

where $\mathbf{L}_{\mathbf{x}}$ has been defined in 34 and ${ }^{o} \mathbf{V}_{e}$ is the spatial motion transform matrix from the target frame to the end effector frame. It is a constant matrix as soon as the target is rigidly attached to the end effector.

As in the previous case the visual feature is related to a virtual point ${ }^{c} \mathbf{X}_{R}$ rather directly ${ }^{c} \mathbf{X}$. Let us note again that the depth $Z_{R}$ is obviously unknown.

\section{EXPERIMENTAL RESULTS}

In this section, we consider only the case of a moving mirror. In Section $\mathrm{V}$ we demonstrated that the cases of motionless mirrors are equivalent to a classical visual servoing scheme. Therefore, as this is a well known approach, we do not show results for these cases and we focus on the original problem of a controlled mirror.

\section{A. Experiments with mirror control: experimental setup}

Figure 6 shows the experimental setup. The mirror is mounted on the end-effector of a 6 d.o.f gantry robot. The camera (which is fully calibrated) is static and looks at the mirror and (thanks to the mirror reflection) to the target. The implementation has be done thanks to the ViSP software [11]. The relative position between the camera and the robot base frame is computed thanks to robot odometry and the localization of the mirror wrt the camera using a nonlinear pose estimation method [12] using four points initially located on the mirror. This is done in an off-line calibration step to compute the constant matrix ${ }^{c} \mathbf{V}_{\emptyset}$ (see (26)). The dots (see Figure 6 (left)) are then removed. Note that the measure of the mirror parameters (reported, eg, in Figure 9 c) are computed thanks to robot odometry and the knowledge of ${ }^{c} \mathbf{T}_{\emptyset}$ obtained during the off-line calibration step. These values are indeed involved in (12), (13) and thus in (26).

\section{B. Servo on one point}

This first experiment depicts a basic visual servoing task. The reflection of the target has to be seen in the middle of the

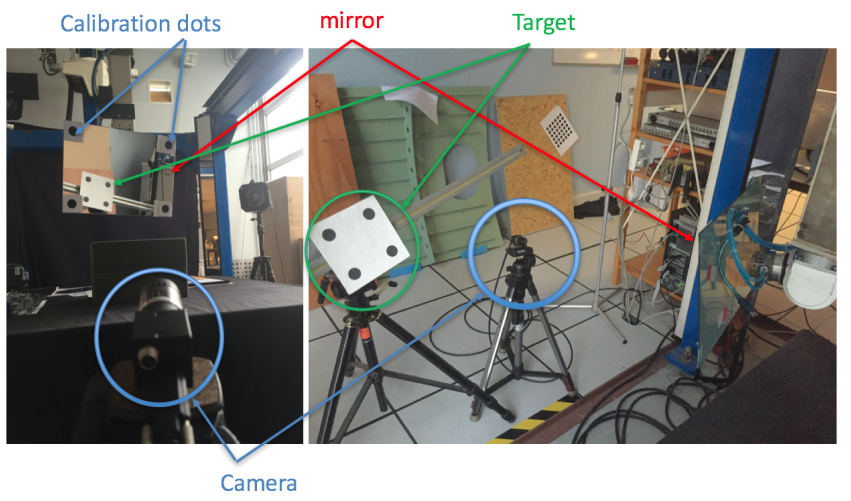

Fig. 6. Experimental setup: the mirror (red arrows) is mounted on the robot end effector; the camera (blue circles) observes the target (green (green) as its reflection in the mirror. The calibration dots are used to determine the position of the camera in the robot reference frame in an off-line calibration step. They are removed for the visual servoing experiments as can be shown in, eg, Figure 7

image (see Figure 7.a) using the control law given by 26. As expected, 3 d.o.f are actually involved to achieve the task (see Figure 7 . c). In this experiment mainly the orientation of the mirror is involved but a small motion along the $\mathrm{Z}$ axis can be also observed (see Figure 7 c). The depth ${ }^{c} Z_{R}$ involved in (10) has been set to $2.5 \mathrm{~m}$, which roughly correspond to the double of the distance between the camera and the mirror. The errors feature an exponential decay (see Figure $7 \mathrm{~b}$ ), which shows the robustness of the control scheme wrt. coarse approximation of the depth ${ }^{c} Z_{R}$.
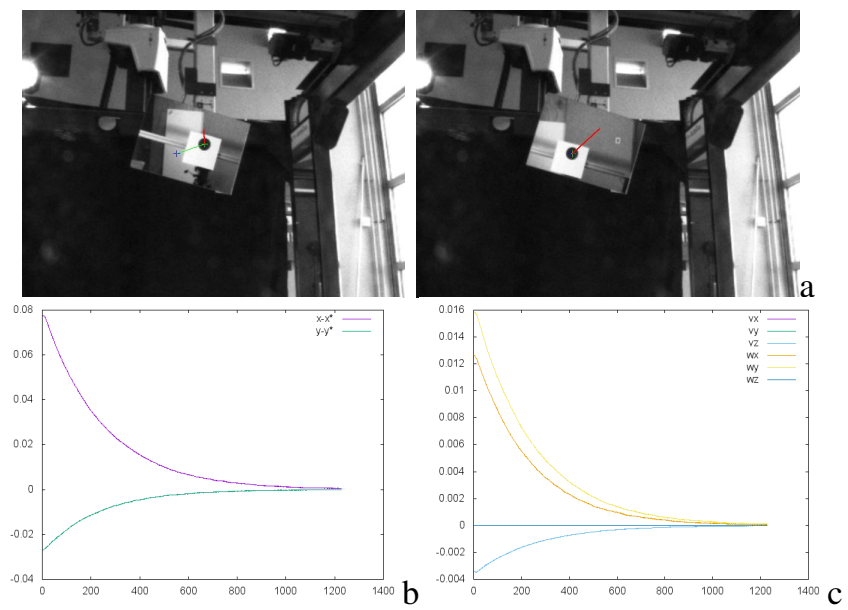

Fig. 7. Servo on one point: (a) initial position (left) and desired (and final) position (right). Video is available through youtube here and in the accompanying video; (b) error $\mathbf{x}-\mathbf{x}^{*}$, (c) mirror velocity in mirror frame $\left({ }^{m} \boldsymbol{v}_{m},{ }^{m} \boldsymbol{\omega}_{m}\right)^{\top}$ in $\mathrm{m} / \mathrm{s}$ and $\mathrm{rad} / \mathrm{s}$. Note that $\left(v_{x}, v_{y}\right.$ and $\omega_{z}$ are always equal to 0 as expected

In the second experiment (Figures 8) we changed the desired position of the point in the upper right of the image. Due to the mirror limited size, it is necessary to use the secondary task defined in Section IV-C in order to avoid the target moving out of the mirror. We see in that case that all the mirror d.o.f are used (see Figure 8.c). 

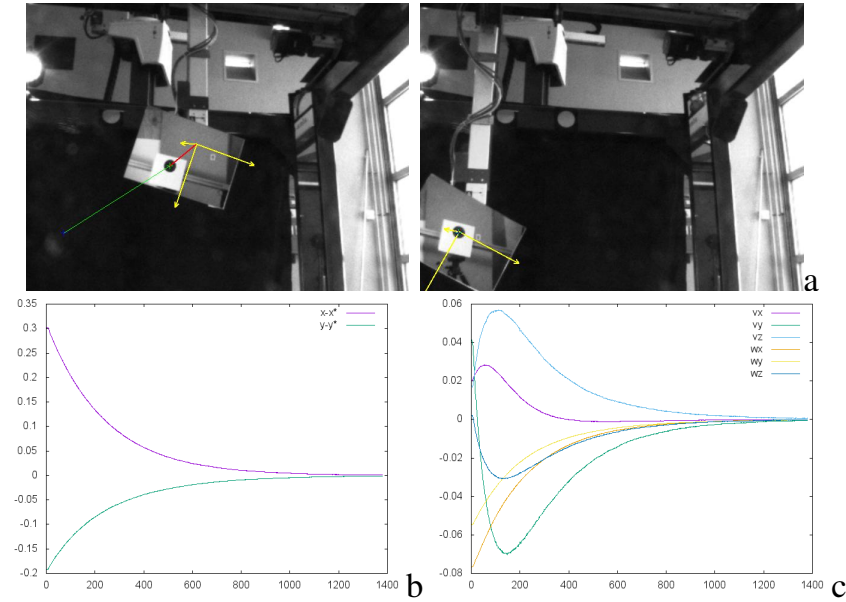

Fig. 8. Servo on one point with large displacement which would induce the target to move out of the mirror. This requires the use of a secondary task as proposed in Section IV-C (a) initial position (left) and desired (and final) position (right). Videos are available through youtube: external view and camera view and in the accompanying video ; (b) error $\mathbf{x}-\mathbf{x}^{*}$, (c) mirror velocities $\left({ }^{m} \boldsymbol{v}_{m},{ }^{m} \boldsymbol{\omega}_{m}\right)$. Note that in that case all the d.o.f. are used to maintain the target in the mirror.

\section{Servo on four points}

In this case we consider a target made of four points. The initial and final (desired) image are shown on Figure 9 As stated in Section IV-B, 3 d.o.f are controlled. Here not only the orientation of the mirror is involved but its depth (in the camera frame) as well. This can be clearly seen on Figure 9, which depicts mirror orientation and depth. The mirror converges toward a desired pose (up to a translation in the mirror planeand a rotation around its normal). The depth ${ }^{c} Z_{R}$ involved in (10) is coarsely set to $4 \mathrm{~m}$. It can be seen on Figure $9 \mathrm{~d}$ than the camera moves backward $50 \mathrm{~cm}$ to reach a desired position. As stated the measure of the mirror parameters (Figure 9 d) are computed thanks to robot odometry and the knowledge of ${ }^{c} \mathbf{T}_{\emptyset}$ obtained during the offline calibration step.

Again due to the fact that rank $\mathbf{J}_{\mathbf{x}}=3$ the target may leave the mirror (which is not of infinite size). This is easy to see on Figure 10 that if the mirror does not move down, the target will no longer be on the mirror at convergence. Here a secondary task is thus considered to maintain the center of gravity (COG) of the target at the COG of the mirror (see Section IV-C]. In that case all the mirror d.o.f are considered to achieve the global task (mainly a translation along $x$ and $y$ in $\mathcal{F}_{m}$ - see Figure 10 ) .

\section{CONCLUSION}

In this paper we investigated visual servoing through mirror reflection and proposed the theoretical background necessary to derive the resulting control law. We demonstrated that when the mirror is static, the problem is very similar to the classical one (with no mirror). In the case where the mirror is moving, we exhibit the analytical form of the interaction matrix and the resulting control law. We demonstrated that, in that case, only 3 d.o.f can be

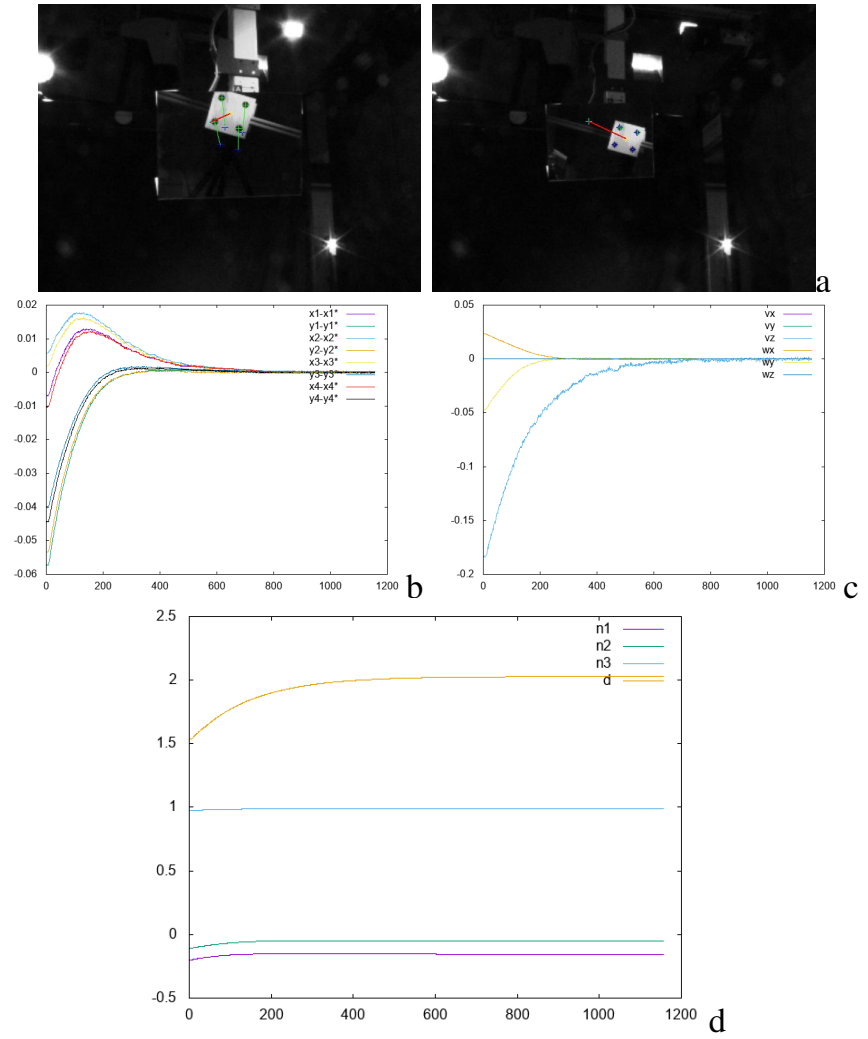

Fig. 9. Servo on 4 points. Camera view: initial position (left) and desired (and final) position on the (right). Videos are available through youtube: external view and camera view and in the accompanying video ; (b) error $\mathbf{x}_{i}-\mathbf{x}_{i}^{*}, i=1 . .4$, (c) mirror velocity in mirror frame $\left({ }^{m} \boldsymbol{v}_{m},{ }^{m} \boldsymbol{\omega}_{m}\right)$, (d) mirror equation $(\mathbf{n}, d)$ with $\mathbf{n}=\left(n_{1}, n_{2}, n_{3}\right)$. Note that only 3 d.o.f are involved to achieve the task (see Section IV-B).

efficiently considered and propose solutions to use efficiently the remaining dof. Experiments has been achieved with a Gantry robot to show the validity of our approach in the case of controlled mirror.

\section{APPENDIX}

\section{A. Proof of equation (6)}

Let us prove that

$$
{ }^{c} \mathbf{X}_{R}=\left(\mathbf{I}-2 \mathbf{n n}{ }^{\top}\right){ }^{c} \mathbf{X}+2 d \mathbf{n}
$$

Proof. Considering (3) and (2)

$$
\begin{aligned}
{ }^{c} \mathbf{X}_{R} & ={ }^{c} \mathbf{R}_{m}{ }^{m} \mathbf{X}_{R}+d \mathbf{n} \\
& ={ }^{c} \mathbf{R}_{m}\left(\mathbf{I}-2{ }^{m} \mathbf{n}^{m} \mathbf{n}^{\top}\right)^{m} \mathbf{X}+d \mathbf{n} \\
& ={ }^{c} \mathbf{R}_{m}\left({ }^{m} \mathbf{R}_{c}{ }^{c} \mathbf{R}_{m}-2^{m} \mathbf{R}_{c} \mathbf{n} \mathbf{n}^{\top} \mathbf{R}_{c}^{\top}\right)^{m} \mathbf{X}+d \mathbf{n} \\
& =\left({ }^{c} \mathbf{R}_{m}-2 \mathbf{n} \mathbf{n}^{\top}{ }^{c} \mathbf{R}_{m}\right)\left({ }^{m} \mathbf{R}_{c}{ }^{c} \mathbf{X}-d^{m} \mathbf{n}\right)+d \mathbf{n} \\
& =\left(\mathbf{I}-2 \mathbf{n} \mathbf{n}^{\top}\right)^{c} \mathbf{R}_{m}\left({ }^{m} \mathbf{R}_{c}{ }^{c} \mathbf{X}-d^{m} \mathbf{R}_{c} \mathbf{n}\right)+d \mathbf{n} \\
& =\left(\mathbf{I}-2 \mathbf{n} \mathbf{n}^{\top}\right)\left({ }^{c} \mathbf{X}-d \mathbf{n}\right)+d \mathbf{n} \\
& =\left(\mathbf{I}-2 \mathbf{n} \mathbf{n}^{\top}\right)^{c} \mathbf{X}-d \mathbf{n}+2 d \mathbf{n} \mathbf{n}^{\top} \mathbf{n}+d \mathbf{n} \\
& =\left(\mathbf{I}-2 \mathbf{n} \mathbf{n}^{\top}\right)^{c} \mathbf{X}+2 d \mathbf{n} \mathbf{n}^{\top} \mathbf{n}
\end{aligned}
$$

$\mathbf{n}$ being a unit vector, $\mathbf{n}^{\top} \mathbf{n}=1$, this leads to equation 5 . 

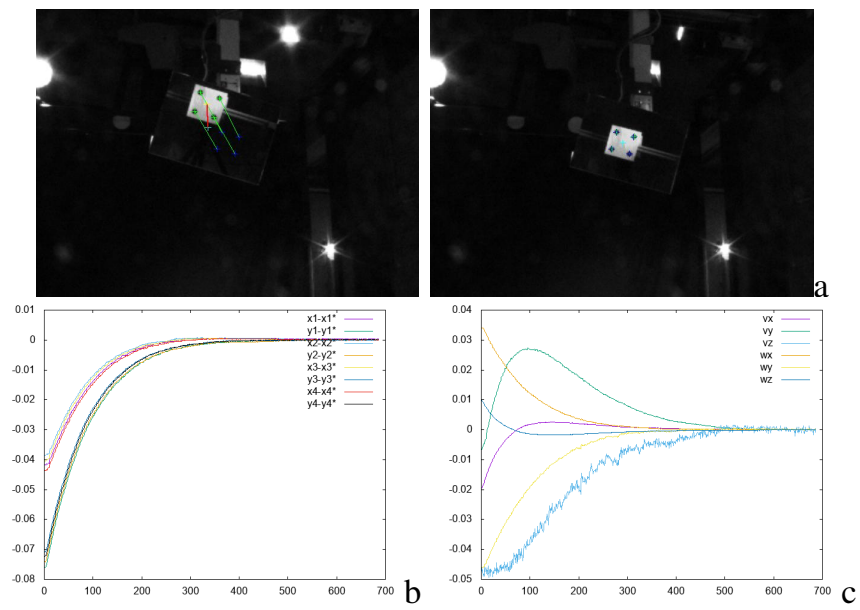

Fig. 10. Servo on 4 points with large displacement with the use of a secondary task as proposed in section IV-C This requires the use of the secondary task proposed in Section IV-C (a) initial position (left) and desired (and final) position (right). Video is available through youtube here and in the accompanying video. Servo on 4 points : (b) error $\mathbf{x}_{i}-\mathbf{x}_{i}^{*}, i=$ $1 . .4$, (c) mirror velocities $\left({ }^{m} \boldsymbol{v}_{m},{ }^{m} \boldsymbol{\omega}_{m}\right)^{\top}$. Note that in that case all the d.o.f. are used to maintain the target in the mirror.

\section{B. Proof of equation (16)}

Let us prove that

$$
{ }^{c} \dot{\mathbf{X}}_{R}=2 \mathbf{n} \dot{d}-2 \dot{\mathbf{n}} d+2\left(\dot{\mathbf{n}} \mathbf{n}^{\top}-\mathbf{n} \dot{\mathbf{n}}^{\top}\right)^{c} \mathbf{X}_{R}
$$

Proof. Injecting equation (15) in (14), we have:

$$
\begin{aligned}
{ }^{c} \dot{\mathbf{X}}_{R}= & 2 \mathbf{n} \dot{d}+2 \dot{\mathbf{n}} d \\
& -2\left(\dot{\mathbf{n}} \mathbf{n}^{\top}+\mathbf{n} \dot{\mathbf{n}}^{\top}\right)\left({ }^{c} \mathbf{X}_{R}-2 \mathbf{n} \mathbf{n}^{\top}{ }^{c} \mathbf{X}_{R}+2 d \mathbf{n}\right) \\
= & 2 \mathbf{n} \dot{d}+2 \dot{\mathbf{n}} d-2\left(\dot{\mathbf{n}} \mathbf{n}^{\top}+\mathbf{n} \dot{\mathbf{n}}^{\top}\right)^{c} \mathbf{X}_{R} \\
& +4\left(\dot{\mathbf{n}} \mathbf{n}^{\top}+\mathbf{n} \dot{\mathbf{n}}^{\top}\right) \mathbf{n} \mathbf{n}^{\top c} \mathbf{X}_{R}-4 d\left(\dot{\mathbf{n}} \mathbf{n}^{\top}+\mathbf{n} \dot{\mathbf{n}}^{\top}\right) \mathbf{n} \\
= & 2 \mathbf{n} \dot{d}+2 \dot{\mathbf{n}} d-2\left(\dot{\mathbf{n}} \mathbf{n}^{\top}+\mathbf{n} \dot{\mathbf{n}}^{\top}\right)^{c} \mathbf{X}_{R} \\
& +4\left(\dot{\mathbf{n}} \mathbf{n}^{\top} \mathbf{n} \mathbf{n}^{\top}+\mathbf{n} \dot{\mathbf{n}}^{\top} \mathbf{n} \mathbf{n}^{\top}\right)^{c} \mathbf{X}_{R} \\
& -4 d\left(\dot{\mathbf{n}} \mathbf{n}^{\top} \mathbf{n}+\mathbf{n} \dot{\mathbf{n}}^{\top} \mathbf{n}\right)
\end{aligned}
$$

note: $\dot{\mathbf{n}}^{\top} \mathbf{n}=0$ since $\dot{\mathbf{n}}=[\mathbf{n}]_{\times} \boldsymbol{\omega}$ leading to $[\mathbf{n}]_{\times} \mathbf{n}=0$

$$
\begin{aligned}
= & 2 \mathbf{n} \dot{d}+2 \dot{\mathbf{n}} d-2\left(\dot{\mathbf{n}} \mathbf{n}^{\top}+\mathbf{n} \dot{\mathbf{n}}^{\top}\right)^{c} \mathbf{X}_{R} \\
& +4\left(\dot{\mathbf{n}} \mathbf{n}^{\top}{ }^{c} \mathbf{X}_{R}\right)-4 d \dot{\mathbf{n}} \\
= & 2 \mathbf{n} \dot{d}-2 \dot{\mathbf{n}} d-2\left(\dot{\mathbf{n}} \mathbf{n}^{\top}+\mathbf{n} \dot{\mathbf{n}}^{\top}\right)^{c} \mathbf{X}_{R}+4\left(\dot{\mathbf{n}} \mathbf{n}^{\top c} \mathbf{X}_{R}\right) \\
= & 2 \mathbf{n} \dot{d}-2 \dot{\mathbf{n}} d+2\left(\dot{\mathbf{n}} \mathbf{n}^{\top}-\mathbf{n} \dot{\mathbf{n}}^{\top}\right)^{c} \mathbf{X}_{R}
\end{aligned}
$$

\section{REFERENCES}

[1] N. Andreff and B. Tamadazte. Laser steering using virtual trifocal visual servoing. The Int. Journal of Robotics Research, 35(6):672694, 2016.

[2] J.P. Barreto, F. Martin, and R. Horaud. Visual servoing/tracking using central catadioptric images. In Int. Symp. on Experimental Robotics, ISER'02, pages 863-869, Bombay, India, July 2002.

[3] F. Chaumette and S. Hutchinson. Visual servoing and visual tracking. In B. Siciliano and O. Khatib, editors, Handbook of Robotics, chapter 24, pages 563-583. Springer, 2008.

[4] F. Chaumette and E. Marchand. A new redundancy-based iterative scheme for avoiding joint limits: application to visual servoing. In IEEE Int. Conf. on Robotics and Automation, ICRA'O0, volume 2, pages 1720-1725, San Francisco, USA, April 2000.
[5] H. Hadj Abdelkader, Y. Mezouar, N. Andreff, and P. Martinet. Imagebased control of mobile robot with central catadioptric cameras. In IEEE Int. Conf. on Robotics and Automation, ICRA'05, pages 35223527, Barcelona, Spain, April 2005.

[6] K. Iida and H. Oku. Saccade mirror 3: High-speed gaze controller with ultra wide gaze control range using triple rotational mirrors. In IEEE Int. Conf. on Robotics and Automation, ICRA'16, pages 624 629, Stockholm, Sweden, May 2016.

[7] K. K. Okumura, H. Oku, and M. Ishikawa. High-speed gaze controller for millisecond-order pan/tilt camera. In IEEE Int. Conf. on Robotics and Automation, ICRA'11, pages 6186-6191, May 2011.

[8] C. Kulpate, M. Mehrandezh, and R. Paranjape. An eye-to-hand visual servoing structure for $3 \mathrm{~d}$ positioning of a robotic arm using one camera and a flat mirror. In IEEE/RSJ Int. Conf. on Intelligent Robots and Systems, IROS'2005, pages 1464-1470, Edmonton, Canada, August 2005

[9] C. Kulpate, R. Paranjape, and M. Mehrandezh. Precise 3d positioning of a robotic arm using a single camera and a flat mirror. Int. Journal of Optomechatronics, 2(3):205-232, 2008.

[10] E. Marchand and F. Chaumette. Addendum to "visual servoing through mirror reflection". http://hal.inria.fr/hal-01445486, May 2017.

[11] E. Marchand, F. Spindler, and F. Chaumette. ViSP for visual servoing a generic software platform with a wide class of robot control skills. IEEE Robotics and Automation Magazine, 12(4):40-52, December 2005.

[12] E. Marchand, H. Uchiyama, and F. Spindler. Pose estimation for augmented reality: a hands-on survey. IEEE Trans. on Visualization and Computer Graphics, 22(12):2633-2651, December 2016.

[13] G.-L. Mariottini and D. Prattichizzo. Image-based visual servoing with central catadioptric cameras. The International Journal of Robotics Research, 27(1):41-56, January 2008.

[14] G.-L. Mariottini, S. Scheggi, F. Morbidi, and D. Prattichizzo. Planar catadioptric stereo: single and multi-view geometry for calibration and localization. In IEEE Int. Conf. on Robotics and Automation, ICRA'09, pages 1510-1515, Kobe, Japan, 2009.

[15] M. Mikawa, K. Yoshida, M. Kubota, and T. Morimitsu. Visual servoing for micro mass axis alignment device. In IEEE/RSJ Int. Conf. on Intelligent Robots and Systems, IROS'96, pages 1091-1096, Osaka, Japan, November 1996.

[16] J. Pagès, C. Collewet, F. Chaumette, and J. Salvi. Visual servoing by means of structured light for plane-to-plane positioning. Technical Report 5579, INRIA, May 2005.

[17] A. Remazeilles, N. Mansard, and F. Chaumette. Qualitative visual servoing: Application to the visibility constraint. In IEEE/RSJ Int. Conf. on Intelligent Robots and Systems, IROS'06, pages 4297-4303, Beijing, China, October 2006.

[18] O. Tahri, H. Araujo, F. Chaumette, and Y. Mezouar. Robust imagebased visual servoing using invariant visual information. Robotics and Autonomous Systems, 61(12):1588-1600, December 2013.

[19] B. Tamadazte and N. Andreff. Weakly calibrated stereoscopic visual servoing for laser steering: Application to phonomicrosurgery. In IEEE/RSJ Int. Conf. on Intelligent Robots and Systems, IROS'14, pages 743-748, Chicago, IL, 2014.

[20] J.-P. Urban, G. Motyl, and J. Gallice. Real-time visual servoing using controlled illumination. The International Journal of Robotics Research, 13(1):93-100, February 1994. 